\title{
Culture-independent genomic characterisation of Candidatus Chlamydia sanzinia, a novel uncultivated bacterium infecting snakes
}

\author{
Alyce Taylor-Brown ${ }^{1}$, Nathan L. Bachmann ${ }^{1}$, Nicole Borel ${ }^{2}$ and Adam Polkinghorne ${ }^{1 *}$
}

\begin{abstract}
Background: Recent molecular studies have revealed considerably more diversity in the phylum Chlamydiae than was previously thought. Evidence is growing that many of these novel chlamydiae may be important pathogens in humans and animals. A significant barrier to characterising these novel chlamydiae is the requirement for culturing. We recently identified a range of novel uncultured chlamydiae in captive snakes in Switzerland, however, nothing is known about their biology. Using a metagenomics approach, the aim of this study was to characterise the genome of a novel chlamydial taxon from the choana of a captive snake. In doing so, we propose a new candidate species in the genus Chlamydia (Candidatus Chlamydia sanzinia) and reveal new information about the biological diversity of this important group of pathogens.
\end{abstract}

Results: We identified two chlamydial genomic contigs: a 1,113,073 bp contig, and a 7,504 bp contig, representing the chromosome and plasmid of Ca. Chlamydia sanzinia strain 2742-308, respectively. The 998 predicted coding regions include an expanded repertoire of outer membrane proteins (Pmps and Omps), some of which exhibited frameshift mutations, as well as several chlamydial virulence factors such as the translocating actin-recruitment phosphoprotein (Tarp) and macrophage inhibition potentiator (Mip). A suite of putative inclusion membrane proteins were also predicted. Notably, no evidence of a traditional chlamydial plasticity zone was identified. Phylogenetically, Ca. Chlamydia sanzinia forms a clade with C. pneumoniae and C. pecorum, distinct from former "Chlamydophila" species.

Conclusions: Genomic characterisation of a novel uncultured chlamydiae from the first reptilian host has expanded our understanding of the diversity and biology of a genus that was thought to be the most well-characterised in this unique phylum. It is anticipated that this method will be suitable for characterisation of other novel chlamydiae.

Keywords: Chlamydia, Culture-independent sequencing, Genomics, Reptile

\section{Background}

The Chlamydiae are a phylum of intracellular bacteria that are characterised by their unique biphasic lifecycle [1-3]. While they are ubiquitous in the environment [4], a significant number are also associated with disease in a wide range of hosts [2]. The traditional family in this phylum, Chlamydiaceae, consists of a single genus, Chlamydia, and includes important human and animal

\footnotetext{
*Correspondence: apolking@usc.edu.au

${ }^{1}$ Centre for Animal Health Innovation, Faculty of Science, Health, Education and Engineering, University of the Sunshine Coast, Sippy Downs, QLD 4556, Australia

Full list of author information is available at the end of the article
}

pathogens such as Chlamydia trachomatis and Chlamydia psittaci. Even though this is the best-understood family in the phylum, two new species, and one Candidatus species, in the genus Chlamydia, were recently described from birds $[5,6]$, highlighting how little we still know about the full diversity of these obligate intracellular pathogens. Aside from human and mammalian hosts [7], chlamydiosis has been reported in both free-ranging and captive reptilian hosts including several snake species, turtles, tortoises and crocodiles, among others [8-11]. The most common species found in these hosts to date is Chlamydia pneumoniae, however, a recent study also 
revealed 16S rRNA sequences corresponding to potentially novel Chlamydia species [11].

Barriers for characterising the biology of these unique intracellular parasites lie in the fact they require a host cell to undergo replication. For many novel species, in vitro culture systems are not available. With deep sequencing becoming faster, more affordable and higher throughput [12], groups within the chlamydia field have recently developed several methods of deep sequencing from clinical samples to gain insight into the biology of these species [13-17]. These alternative methods bypass the labour-intensive and costly culture step that has hampered genomic characterisation of novel pathogens.

In the current study, we have utilised a cultureindependent method to sequence the genome of a previously uncharacterised and uncultivable new member of the genus Chlamydia. The subsequent comparative genomics enabled by this approach allows for fast and effective identification of a number of hallmarks of chlamydial biology in these novel species, including virulence factors and membrane proteins.

\section{Results and discussion}

\section{Metagenome reconstruction and chlamydial genome} assembly

$5,561,445$ paired reads were obtained from the treated DNA sample following shotgun sequencing on an Illumina MiSeq. As no reference genome was available for this putative novel species, reads were trimmed prior to de novo assembly into 261,306 contigs. BLASTn analysis revealed two contigs that were suspected to be of chlamydial origin: a 1,113,073 bp chromosomal contig with $81 \%$ nucleotide identity with C. pneumoniae LPCoLN (CP001713.1), and a 7,504 bp plasmid contig with $77 \%$ nucleotide identity with the plasmid of C. pneumoniae LPCoLN (CP001714.1) (Additional file 1: Table S1). 329,886 reads mapped to the chromosomal contig, accounting for $\sim 6 \%$ of the reads (Additional file 1: Table S2). The mean read coverage across the genome was $\sim 44 \times$, with at least $10 \mathrm{x}$ read coverage at every base with the exception of some of the predicted polymorphic membrane proteins. Interestingly, the mean coverage of the plasmid was estimated to be $\sim 1888 \mathrm{x}$, with the plasmid reads accounting for $\sim 37 \%$ of the total reads.

Despite treatment of the DNA to enrich for microbial DNA, at least 227,252 contigs are believed to be hostderived, based on BLASTn analysis against two available snake genomes (Python bivittatus; AEQU00000000.2 and Pantherophis guttatus; JTLQ00000000.1), although the majority of these contigs are very short (Additional file 1: Table S2, Table S3). While the method used to enrich for microbial DNA relies on depletion of methylated DNA, there is evidence to suggest that vertebrate mitochondrial DNA may not be methylated at many regions [18]. This would explain the high coverage $(\sim 1904 \times)$ observed for the mitochondrial genome obtained in this metagenome, accounting for $\sim 5 \%$ of the reads. (Additional file 1: Table S2).

The metagenome was also screened for host-associated microflora to assess the proportion of reads devoted to other bacterial species. Full or partial 16S rRNA sequences were detected for five non-chlamydial bacteria in the sample, with significantly lower coverage than the chlamydial genome. (Additional file 1: Table S4).

Automated annotation of the chlamydial chromosome and plasmid by RAST followed by manual annotation in Artemis resulted in prediction of 998 coding regions, demonstrating a similarly reduced gene content to other members of the Chlamydiaceae (Table 1). Roughly one third of the genome consists of hypothetical proteins (314). The two chlamydial genomic contigs of uncultured Chlamydia sp. 2742-308, had G + C contents of $38.5 \%$ and $32.3 \%$, respectively (Table 1 ) $[5,6]$. The chromosome was shown to be able to be circularised in silico by read mapping across the contig break (Additional file 2: Figure S1).

\section{Description of "Candidatus Chlamydia sanzinia", sp. nov}

Based on the novel nature of the assembled chlamydial genome sequenced in this sample, we propose for it a novel species with Candidatus status in the genus Chlamydia, in the absence of maintenance in culture: Ca. Chlamydia sanzinia (Sanzinia, pertaining to the host genus name). $C a$. C. sanzinia shares $<97 \% 16 S$ rRNA nucleotide identity with other Chlamydia spp. Additionally, it shares $<96 \%,<98 \%,<96 \%,<95 \%$ and $<95 \%$ nucleotide sequence identity with other Chlamydia spp. for rpoN, ftsK, pepF, adk and hemL, respectively (Additional file 1: Table S5); classifying it as a new species using the scheme recommended by Pillonel et al. [19]. This novel species was detected in the choana of a captive asymptomatic Madagascar tree boa (Sanzinia madagascariensis volontany) in a private collection in Switzerland.

\section{Ca. Chlamydia sanzinia forms a distinct clade with C. pneumoniae and C. pecorum}

Using the novel sequence data for this unique chlamydial taxon, we extracted the core genome for comparative phylogenetic analyses to show that $\mathrm{Ca}$. Chlamydia sanzinia is most closely related to C. pneumoniae (LPCoLN strain), with an average nucleotide identity of $76.9 \%$, in agreement with the 16S rRNA sequence identity previously described for this putative novel species [11]. In our phylogenetic tree construction, this taxon shares the same minor clade with C. pneumoniae and C. pecorum, within a major clade comprised of former "Chlamydophila" spp. and the novel avian Chlamydia spp and distinct from C. muridarum, C. suis and C. trachomatis (Fig. 1). Branch lengths indicate that $C a$. C. sanzinia and $C$. 
Table 1 Comparative analysis of chlamydial genome features. One strain representative for each species was analysed

\begin{tabular}{|c|c|c|c|c|c|c|c|c|c|c|c|c|c|}
\hline $\begin{array}{l}\text { Species, } \\
\text { strain (accession } \\
\text { number) }\end{array}$ & $\begin{array}{l}\text { Ca. } \\
\text { C. sanzinia } \\
\text { 2742-308 } \\
(\text { CP014639) }\end{array}$ & $\begin{array}{l}\text { C. pneumoniae } \\
\text { LPCoLN } \\
\text { (CP006571.1) }\end{array}$ & $\begin{array}{l}\text { C. pecorum } \\
\text { MC/MarsBar } \\
\text { (NZ_CM002310.1) }\end{array}$ & $\begin{array}{l}\text { C. psittaci 6BC } \\
\text { (NC_017287.1) }\end{array}$ & $\begin{array}{l}\text { C. felis } \\
\text { Fe/C-56 } \\
\text { (AP006861.1) }\end{array}$ & $\begin{array}{l}\text { C. caviae } \\
\text { GPIC } \\
\text { (AE015925.1) }\end{array}$ & $\begin{array}{l}\text { C. abortus } \\
\text { S26/3 } \\
\text { (NC_004552.2) }\end{array}$ & $\begin{array}{l}\text { C. avium } \\
\text { 10DC88 } \\
\text { (CP006571.1) }\end{array}$ & $\begin{array}{l}\text { C. gallinacea } \\
08-1274 / 3 \\
\text { (CP015840.1) }\end{array}$ & $\begin{array}{l}\text { Ca. C. ibidis } \\
\text { 10-1398/6 } \\
\text { (APJW00000000.1) }\end{array}$ & $\begin{array}{l}\text { C. muridarum } \\
\text { Nigg } \\
\text { (NC_002620.2) }\end{array}$ & $\begin{array}{l}\text { C. trachomatis } \\
\text { A/HAR-13 } \\
\text { (NC_007429.1) }\end{array}$ & $\begin{array}{l}\text { C. suis } \\
\text { MD56 } \\
\text { (CM002267.1) }\end{array}$ \\
\hline $\begin{array}{l}\text { Chromosome } \\
\text { length (Mbp) }\end{array}$ & 1.11 & 1.24 & 1.11 & 1.17 & 1.17 & 1.17 & 1.14 & 1.04 & 1.05 & 1.15 & 1.07 & 1.04 & 1.07 \\
\hline $\begin{array}{l}\text { GC } \\
\text { content (\%) }\end{array}$ & 38.5 & 40.5 & 41.1 & 39.1 & 39.4 & 39.2 & 39.9 & 36.9 & 37.9 & 38.3 & 40.3 & 41.3 & 42.0 \\
\hline No. CDSs & 998 & 1097 & 945 & 1003 & 1005 & 998 & 964 & 940 & 898 & 949 & 904 & 911 & 915 \\
\hline $\begin{array}{l}\text { Hypothetical } \\
\text { proteins }\end{array}$ & 314 & 426 & 297 & 337 & 324 & 376 & 219 & 242 & 207 & 235 & 353 & 294 & 218 \\
\hline $\begin{array}{l}\text { Plasmid } \\
\text { length (Kbp) } \\
\text { (No. ORFS) }\end{array}$ & $7.5(8)$ & $7.5^{\mathrm{a}}(8)$ & $7.5^{\mathrm{a}}(8)$ & $7.5(8)$ & $7.5(8)$ & $7.9(7)$ & $\mathrm{Np}$ & $7.1(7)$ & $7.0(7)$ & $\mathrm{Np}$ & $7.5(8)$ & $7.5^{\mathrm{b}}(8)$ & $5.9(6)$ \\
\hline
\end{tabular}

Np No plasmid

a Plasmid not present in all strains

bPlasmid length of C. trachomatis L2b/CS784/08 


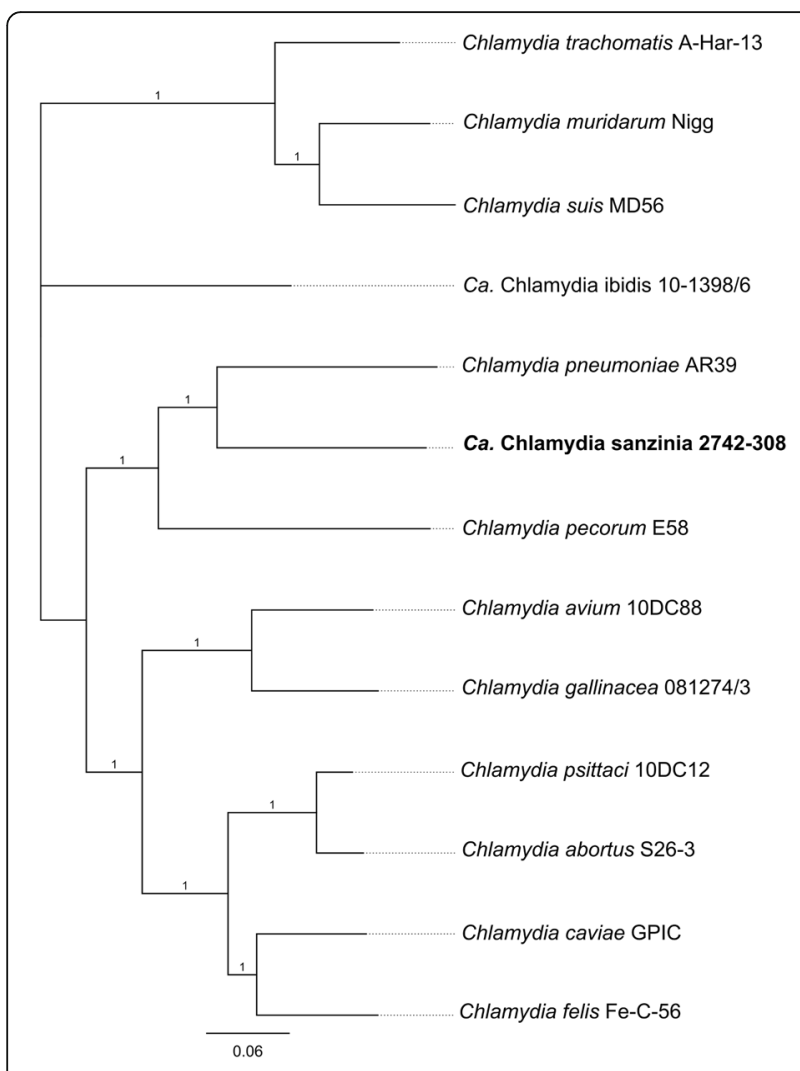

Fig. 1 Chlamydia species core genome phylogenetic tree. The core genome was extracted using the LS-BSR package and phylogenetic tree constructed using FastTree. Numbers on the branches indicate support values

pneumoniae have evolved at a similar rate after divergence from a common ancestor.

\section{The genome of $\mathrm{Ca}$. Chlamydia sanzinia harbours a 7.5 Kbp cryptic plasmid}

Chlamydial plasmids are nonconjugative, nonintegrative and found in most chlamydial species [19]. While we are still understanding the function of this plasmid, studies have suggested roles in regulation of the developmental cycle and in influencing tissue tropism and disease outcome [20-22]. De novo assembly of the trimmed reads produced an extra-chromosomal contig resembling the chlamydial plasmid, the gene content and arrangement of which are typical of other chlamydial plasmids (Fig. 2b). The $C a$. C. sanzinia plasmid proteins share 42.7 to 87.2 $\%$ amino acid similarity with Chlamydia spp. plasmid proteins (Additional file 3: Tables S6-S13). The phylogenetic tree constructed from a concatenated alignment of the conserved proteins, reflects these relationships (Fig. 2a), which mirror that of the chromosome. The nucleotide sequence identity across the plasmid was much lower, ranging from 31 to $69 \%$, although much of this variation can be attributed to genes absent from some of the plasmids (Table 1, Fig. 2b).

It is also interesting to note that coverage of the plasmid contig was almost 43 times that of the chromosome ( $\sim 1,888 \times$ vs $\sim 44 \times$ and accounting for $\sim 37 \%$ of the reads), suggesting a plasmid copy number of up to 43 per chromosome. This seems unlikely given previous descriptions of plasmid copy numbers in the range of two to ten [23-25]. An alternative explanation for the high coverage is that the MDA process preferentially amplifies the plasmid. Other studies have also shown that plasmid copy numbers change throughout the developmental cycle [23-25].

\section{The genome of $\mathrm{Ca}$. Chlamydia sanzinia does not appear to contain a plasticity zone}

The chlamydial plasticity zone (PZ) is a region of extensive variation between chlamydial genomes [2, 7], which, while highly variable, generally harbours (i) acetyl coA carboxylase chains (accBC), (ii) cytotoxin genes/adherence factor, (iii) phospholipase D (PLD), (iv) membrane attack complex/perforin (MACPF), (v) tryptophan biosynthesis operon (trpABFCDR, kynU, prsA) and (vi) purine biosynthesis genes (guaAB-add) [2, 7]. Iterations of the chlamydial PZ have been described in the genomes of all species of Chlamydia to date ranging from $\sim 12 \mathrm{kbp}$ to $\sim 86 \mathrm{kbp}$, with 11 to 48 genes $[2,7]$.

Despite rigorous homology and conserved domain searches through all 261,306 contigs, the only features of a chlamydial $\mathrm{PZ}$ in $\mathrm{Ca}$. C. sanzinia are $a c c \mathrm{~B} \&$ acc $\mathrm{C}$ (Cs308_0799 \& Cs308_0800) (Fig. 3). While this region is very small, it is similar to that of its closest relative, $C$. pneumoniae LPCoLN and to that of C. avium 10DC88, both of which only possess accBC and MACPF. Two hypothetical proteins have weak sequence similarity to C. psittaci adherence factor (tox) (Cs308_0802 and Cs308_0803). The finding of neither a MACPF or plasticity zone-PLD is consistent given the evidence that MACPF may assist PLDs in lipid acquisition and processing [2], in which case the absence of a MACPF may depend on the absence of a PLD. The lack of a complete trp operon, a feature shared with its closest relative, $C$. pneumoniae, as well as other Chlamydia spp. except $C$. pecorum, C. caviae and C. felis, suggests a different pathway for synthesising tryptophan. Interestingly, an aromatic amino acid synthase, which has been described as an alternative pathway for trp synthesis in C. pneumoniae [26], also appears to be absent. The lack or truncation of the guaAB-add operon for purine biosynthesis is common to C. trachomatis, C. abortus, and some strains of C. pneumoniae and $C$. psittaci. The genome of $\mathrm{Ca}$. C. sanzinia does however appear to possess an AMP nucleosidase (Cs308_0522) which plays a role in purine nucleoside salvage. 


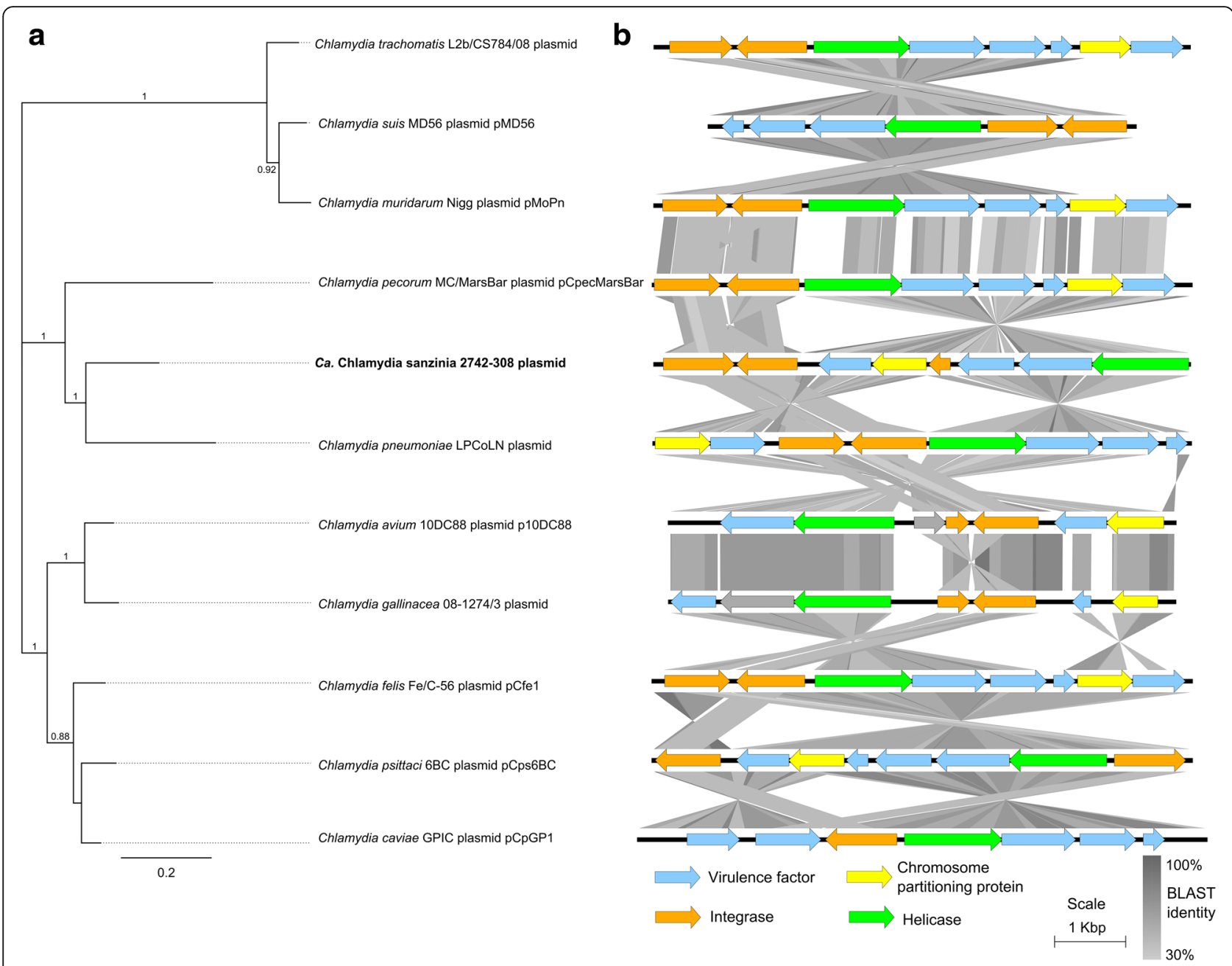

Fig. 2 Chlamydial plasmid phylogeny and arrangement. (a) Chlamydial plasmid proteins were extracted from each sequence, concatenated and aligned prior to phylogenetic tree construction using the FastTree algorithm in Geneious; (b) Chlamydia plasmid nucleotide sequences were compared via tBLASTx analysis and their arrangement plotted in EasyFig Block arrows represent proteins, coded by colour and grey shading represents sequence homology

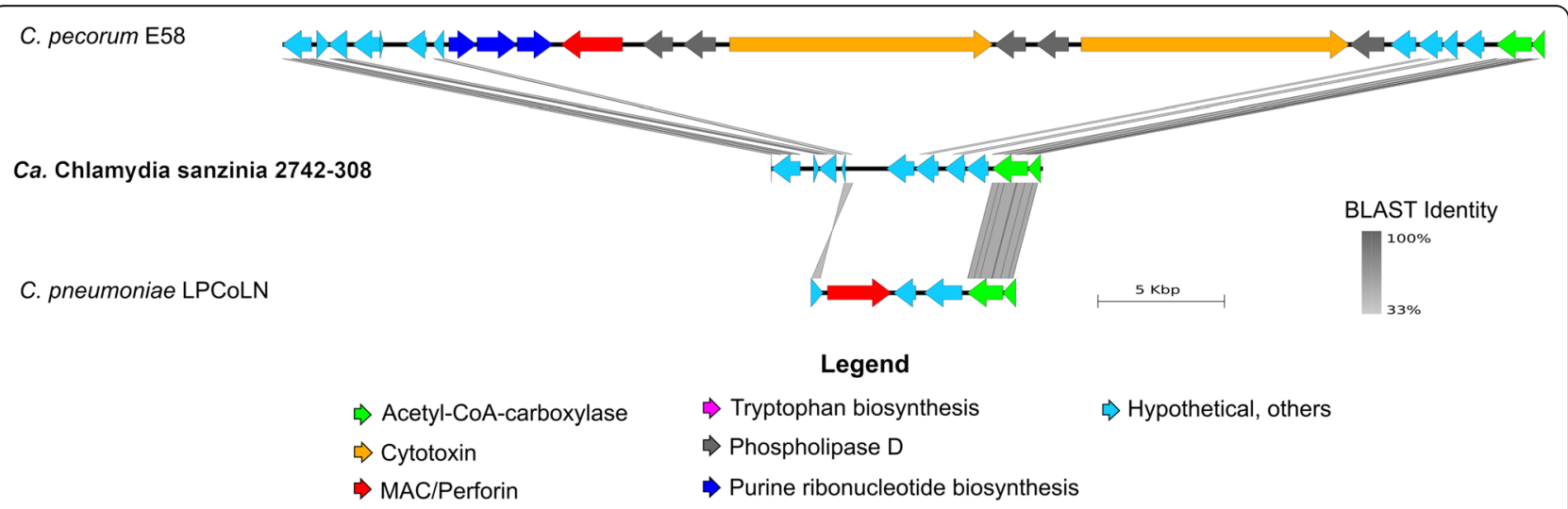

Fig. 3 Lack of the plasticity zone in Ca. Chlamydia sanzinia. The region of the genome encoding the plasticity zone in C. pecorum and C. pneumoniae were compared to that of $\mathrm{Ca}$. C. sanzinia via tBLASTx analysis and their arrangement plotted in EasyFig. Block arrows represent proteins, coded by colour and grey shading represents sequence homology 
We considered the possibility that the plasticity zone was either not sequenced or not assembled, given that multiple displacement amplification may introduce an amplification bias [27], as seen for the plasmid. As shown in Additional file 2: Figure S1, however, overlapping read mapping to the joined ends of the chromosomal contig demonstrates that we have obtained the complete genome for this bacterium.

The absence of an obvious chlamydial plasticity zone characteristic to other members in the genus, as such, sets this putative novel species apart from its known closest phylogenetic relatives. Strain diversity studies in other chlamydial species suggest that further investigation could elucidate remnants of a PZ if other strains of this novel taxon were to be characterised.

\section{An expanded repertoire of polymorphic membrane proteins are located on "pmp islands" for $\mathrm{Ca}$. Chlamydia sanzinia}

Chlamydial membrane proteins are thought to play an important role in host-parasite interaction throughout the chlamydial developmental cycle. Given their antigenic properties, many have also been the target of extensive vaccine studies [28]. Polymorphic membrane proteins (Pmps) are one such family of membrane protein, unique to chlamydiae, that are highly variable but are united by their GGA (I, L, V) and FxxN tetrapeptide motifs [28, 29]. They also function as autotransporters in the Type $\mathrm{V}$ secretion system [29]. Investigation of the genome of $\mathrm{Ca}$. C. sanzinia revealed not only the presence of homologues of previously described pmp genes, exhibiting the aforementioned motifs, but also the presence of an expanded group of membrane proteins annotated as omp5, an outer membrane protein (Cs308_0070, 0072, 0079, 0084, 0085, Cs308_674677). These protein encoding genes share significant sequence homology with either $p m p$ or omp proteins from C. pneumoniae, C. pecorum, C. psittaci and $C$. abortus (43-62\% amino acid similarity). This family of membrane protein genes, together with the pmps, are arranged in four clusters, not dissimilar to the $p m p$ distribution in other chlamydial genomes (Additional file 4: Figure S2).

Although twelve omp5 genes were annotated by automated methods, further analysis determined three of these (Cs308_0074, Cs308_0076 and Cs38_0077) to be fragments of two pmpG pseudogenes (Cs308_0073 and Cs308_0075), with truncations attributed to frameshift mutations (Additional file 4: Figure S3). The fragments together contain the repeat motifs, middle domain protein and autotransporter domains characteristic to pmps. Frameshifts are common to $p m p$ encoding genes, and it has been suggested that these mutations promote antigenic diversity [26].
Pmp genes account for around 4 to $5 \%$ of the coding regions in closely related chlamydial species (up to $22 \%$ for some strains of $C$. pneumoniae [26]). The predicted Pmp-encoding genes in this genome account for $3.1 \%$; with the inclusion of the additional omp5 genes these membrane proteins together account for approximately $5 \%$ of the coding regions of this genome, suggesting the expanded $p m p$ and $o m p$ repertoire confers characteristics advantageous to survival inside the host cells infected by this novel chlamydial agent.

\section{Ca. Chlamydia sanzinia displays genomic hallmarks of a pathogen}

The Chlamydiae possess a number of mechanisms to exploit the host cell machinery in order to survive and replicate inside a host cell. One of the ways in which chlamydiae achieve this is by the secretion of "effectors", virulence factors which function to influence host signalling, cleave host proteins and suppress host defences. Homologues of several virulence factors secreted by the Type Three Secretion System (T3SS) are present in the genome of $\mathrm{Ca}$. C. sanzinia: namely, a homolog of translocated actin-recruiting phosphoprotein (Tarp) (Cs308_0200), macrophage inhibition potentiator (mip) (Cs308_0291), tail-specific protease (tsp) (Cs308_0179) and serine/threonine protein kinase (PknD) (Cs308_0709) [30], while genes encoding the chlamydial outer protein, CopB, and a chlamydial protein associating with death domains (CADD), appear to be absent [30]. Another chlamydial virulence factor, chlamydial protease-like activity factor (CPAF) (Cs308_0639), secreted by the sec-dependent pathway [31], was also identified in this novel genome.

The inclusion membrane proteins (Incs) are another group of membrane proteins unique to the Chlamydiae, exposed to the host cytosol and hypothesised to be involved in inclusion membrane biogenesis [32, 33]. They share little sequence similarity but instead possess a 40-60 amino acid bi-lobed hydrophobic structure [32]. In addition to two copies of IncA and single copy each of IncB and IncC (Cs308_0059, Cs308_0863, Cs_0864, Cs_0885), an additional 41 putative Inc proteins were predicted in silico in the $\mathrm{Ca}$. C. sanzinia genome (Additional file 5: Table S14). This is within the range described for other Chlamydia spp, which are predicted to encode as few as 36 Inc proteins (C. trachomatis) or up to as many as 107 (C. pneumoniae) [32], providing further evidence that these proteins are integral to inclusion development and host interaction across the genus [2].

\section{Conclusion}

For many novel species within and outside of the Chlamydiae, culture-independent sequencing from clinical samples provides a unique opportunity to understand 
the biology of the species for which there is a) no established culture system or b) no reference genome. While our group and others have recently used several genome sequencing methods to broaden our knowledge of previously described species in the genus Chlamydia [13-17], the current study suggests that a shotgun deep sequencing approach is better suited to novel species. For instance, using "bait" probes designed from a reference genome risks overlooking previously undetected or undescribed features, such as a plasmid [25]. Likewise, using only homology or binning approaches for metagenomes from clinical samples such as this may also omit accessory proteins or extrachromosomal sequences that either share no homology to known proteins or are lacking in conserved phylogenetic markers. The use of MDA for whole genome amplification prior to genome sequencing does come with its own limitations however, such as preferential amplification of certain genomic regions or particular bacteria in a microbial community [27, 34]. The amplification skew is unpredictable and appears to be heavily dependent on both the complexity of the sample and the MDA protocol applied [27]. These issues appear to be able to be partially overcome by combining MDA with other depletion or enrichment methods [15]. Nonetheless, these tools are particularly useful for uncultivable intracellular organisms such as members of the phylum Chlamydiae, and can likely be incredibly valuable for further characterisation of a range of novel chlamydiae reported within and outside the genus Chlamydia. This method also circumvents sequencing of genetic changes that are often acquired during passaging.

We have used this method to sequence and characterise a novel uncultured bacterium in the genus Chlamydia from the first reptilian host, expanding our understanding of the diversity and biology of a genus that was thought to be the most well-characterised in this biologically unique phylum.

\section{Methods}

DNA extraction and microbial DNA enrichment

Genomic DNA was extracted from a swab from the choana of a Madagascar tree boa (Sanzinia madagascariensis volontany) as per [11]. Total genomic DNA was then subject to host methylated-DNA depletion using the NEBNext Microbiome DNA Enrichment kit (New England BioLabs, USA), according to manufacturer's instructions. The selectively enriched DNA was purified by ethanol precipitation before being subject to multiple displacement amplification using the Qiagen Repli-G mini kit (Qiagen, Germany), according to manufacturer's instructions.

\section{Genome sequencing and assembly}

Sequencing was carried out on an Illumina MiSeq at the Australian Genome Research Facility, Walter \& Eliza Hall
Institute, Parkville, Australia using 150 bp paired end reads. Read quality was assessed with FastQC v0.11.2 and trimmed using Trimmomatic v.035 [35]. Trimmed reads were de novo assembled using SPAdes v3.1.1 [36], with kmer values of 51, 71, 91, 101 and 127 in both multi-cell and single-cell mode. QUAST [37] was used to assess the quality of the assemblies (Additional file 6: Table S15).

\section{Genome annotation and analysis}

Resulting contigs were subject to a BLASTx search against an in-house chlamydial protein database. Contigs longer than $1000 \mathrm{bp}$ with hits with e-values $\leq 0.005$ and identity values $\geq 60 \%$ were subsequently also manually compared to the NCBI database. Two chlamydial contigs were uploaded to RAST [38] for automated annotation. Additional coding regions were identified and annotated using Artemis [39]. The chromosomal contig was split to resemble the genome architecture of $C$. trachomatis, and reads were mapped back to the assembly to assess genome coverage. A 60 bp region with $<10 x$ coverage was removed from the 5 ' end of the contig, resulting in reads overlapping the the 5' and 3' ends of the contig. A similar method was also applied to the plasmid contig; a $124 \mathrm{bp}$ region was removed from the 5' end. The nucleotide sequence of the genome of uncultured $\mathrm{Ca}$. Chlamydia sanzinia strain 2742-308 was deposited in Genbank under accession numbers CP014639 (chromosome) and CP014640 (plasmid).

To identify the origins of non-chlamydial contigs, Metaxa [40] was employed to detect ribosomal RNA sequences of prokaryotic and eukaryotic origins. These results informed downstream read-mapping to assess the proportion of reads belonging to different origins using Geneious [41], BWA aligner [42], and BEDTools [43]. The visualisation of the BLAST comparisons in Figs. 2 and 3 were generated using Easyfig [44].

\section{Phylogenetic analysis}

To assess the genome-wide phylogenetic relationships among Chlamydia spp., the core genome was extracted using the LS-BSR package [45]. A phylogenetic tree was constructed from the computed alignment using FastTree [46] and visualised in Geneious [41]. For the plasmid phylogeny, each gene was extracted and translated in silico, prior to concatenation. The phylogenetic tree was then constructed from the resulting alignment.

\section{Inclusion membrane protein prediction}

The amino acid sequences for all 314 hypothetical proteins were subject to transmembrane helix prediction using TMHMM [47]. Hydropathy plots were visualised to identify characteristic bi-lobed hydrophobic domains. 


\section{Additional files}

Additional file 1: Table S2. Chlamydial contig BLAST analysis. Table S3 Metagenome coverage statistics. Table S4. Putative host contig analysis. Table S5. rRNA-containing contigs detected using Metaxa. Table S5. Phylogenetic marker gene BLAST analysis. (PDF $152 \mathrm{~kb}$ )

Additional file 2: Figure S1. Contig break mapping. The chromosomal contig was split to resemble the genome architecture of $C$. trachomatis, and reads were mapped back to the assembly to assess genome coverage in Geneious. A 60 bp region with $<10 \times$ coverage was removed from the $5^{\prime}$ end of the contig, resulting in reads overlapping the the $5^{\prime}$ and $3^{\prime}$ ends of the contig. (JPG $257 \mathrm{~kb}$ )

Additional file 3: Tables S6-13. Plasmid protein homology. (XLSX 22 kb) Additional file 4: Figure S2. Locations of "pmp islands" in the uncultured Ca. Chlamydia sanzinia genome and selected chlamydial genomes. Figure was constructed in Geneious. Figure S3. Schematic representation of pmp pseudogenes attributed to frameshift mutations. Open reading frames are coloured blue in frame and genes/pseudogenes are coloured white on the forward strand. Vertical black lines represent stop codons. Diagonal blue lines represent fragmentation due to premature stop codons. Figure constructed using Artemis. (PDF $140 \mathrm{~kb}$ )

Additional file 5: Table S14. Inclusion membrane prediction. (PDF 99 kb) Additional file 6: Table S15. QUAST report. (PDF $127 \mathrm{~kb}$ )

\section{Acknowledgements}

We thank Simon Rüegg for sample collection Carmen Kaiser for DNA extraction and initial PCR screening. We thank Helena Seth-Smith for her advice on the preparation of our samples for culture-independent genome sequencing as well as downstream analysis.

\section{Availability of data and materials}

The nucleotide sequence of the genome of Ca. Chlamydia sanzinia was deposited in Genbank under accession numbers CP014639 (chromosome) and CP014640 (plasmid); bioproject PRJNA312988.

\section{Authors' contributions}

ATB performed the experiments and analysis, and wrote the main manuscript. NLB contributed to analysis and prepared Fig. 3 and Additional file 4: Figure S2. All authors reviewed the manuscript. All authors read and approved the final manuscript.

\section{Competing interests}

The authors declare no competing financial interests.

\section{Ethics approval and consent to participate}

The collection and molecular analysis of the snake sample was approved and performed in accordance with the relevant guidelines and regulations of the Veterinary Office of Canton Zurich (authorization no. ZH010/15).

\section{Author details}

${ }^{1}$ Centre for Animal Health Innovation, Faculty of Science, Health, Education and Engineering, University of the Sunshine Coast, Sippy Downs, QLD 4556, Australia. ${ }^{2}$ Institute of Veterinary Pathology, University of Zurich,

Winterthurerstrasse 268, 8057 Zurich, Switzerland.

\section{Received: 7 June 2016 Accepted: 27 August 2016}

\section{Published online: 05 September 2016}

\section{References}

1. Abdelrahman YM, Belland RJ. The chlamydial developmental cycle. FEMS microbiology reviews. 2005;29(5):949-59.

2. Nunes A, Gomes JP. Evolution, phylogeny, and molecular epidemiology of Chlamydia. Infect Genet Evol. 2014;23:49-64.

3. Taylor-Brown A, Vaughan L, Greub G, Timms P, Polkinghorne A. Twenty years of research into Chlamydia-like organisms: a revolution in our understanding of the biology and pathogenicity of members of the phylum Chlamydiae. Pathog Dis. 2015;73(1):1-15.
4. Lagkouvardos I, Weinmaier T, Lauro FM, Cavicchioli R, Rattei T, Horn M. Integrating metagenomic and amplicon databases to resolve the phylogenetic and ecological diversity of the Chlamydiae. ISME J. 2014:8(1):115-25.

5. Vorimore F, Hsia RC, Huot-Creasy H, Bastian S, Deruyter L, Passet A, Sachse K, Bavoil P, Myers G, Laroucau K. Isolation of a new Chlamydia species from the Feral Sacred Ibis (Threskiornis aethiopicus): Chlamydia ibidis. PloS One. 2013;8(9):e74823

6. Sachse K, Bavoil PM, Kaltenboeck B, Stephens RS, Kuo CC, Rossello-Mora R, Horn M. Emendation of the family Chlamydiaceae: proposal of a single genus, Chlamydia, to include all currently recognized species. Syst Appl Microbiol. 2015;38(2):99-103.

7. Bachmann NL, Polkinghorne A, Timms P. Chlamydia genomics: providing novel insights into chlamydial biology. Trends in Microbiology. 2014;22(8):464-72.

8. Bodetti TJ, Jacobson E, Wan C, Hafner L, Pospischil A, Rose K, Timms P. Molecular evidence to support the expansion of the hostrange of Chlamydophila pneumoniae to include reptiles as well as humans, horses, koalas and amphibians. Syst Appl Microbiol. 2002;25(1):146-52.

9. Soldati G, Lu ZH, Vaughan L, Polkinghorne A, Zimmermann DR, Huder JB, Pospischil A. Detection of mycobacteria and chlamydiae in granulomatous inflammation of reptiles: a retrospective study. Vet Pathol. 2004;41(4):388-97.

10. Huchzermeyer FW, Langelet E, Putterill JF. An outbreak of chlamydiosis in farmed Indopacific crocodiles (Crocodylus porosus). J S Afr Vet Assoc. 2008; 79(2):99-100

11. Taylor-Brown A, Ruegg S, Polkinghorne A, Borel N. Characterisation of Chlamydia pneumoniae and other novel chlamydial infections in captive snakes. Vet Microbiol. 2015;178(1-2):88-93.

12. Didelot $X$, Bowden R, Wilson DJ, Peto TE, Crook DW. Transforming clinical microbiology with bacterial genome sequencing. Nat Rev Genet. 2012; 13(9):601-12.

13. Putman TE, Suchland RJ, Ivanovitch JD, Rockey DD. Culture-independent sequence analysis of Chlamydia trachomatis in urogenital specimens identifies regions of recombination and in-patient sequence mutations. Microbiology. 2013;159(10):2109-17.

14. Seth-Smith HM, Harris SR, Skilton RJ, Radebe FM, Golparian D, Shipitsyna E, Duy PT, Scott P, Cutcliffe LT, O'Neill C, et al. Whole-genome sequences of Chlamydia trachomatis directly from clinical samples without culture. Genome Res. 2013;23(5):855-66.

15. Seth-Smith HM, Harris SR, Scott P, Parmar S, Marsh P, Unemo M, Clarke IN, Parkhill J, Thomson NR. Generating whole bacterial genome sequences of low-abundance species from complex samples with IMS-MDA. Nat Protoc. 2013;8(12):2404-12.

16. Christiansen MT, Brown AC, Kundu S, Tutill HJ, Williams R, Brown JR, Holdstock J, Holland MJ, Stevenson S, Dave J, et al. Whole-genome enrichment and sequencing of Chlamydia trachomatis directly from clinical samples. BMC Infect Dis. 2014;14:591.

17. Bachmann NL, Sullivan MJ, Jelocnik M, Myers GS, Timms P, Polkinghorne A. Culture-independent genome sequencing of clinical samples reveals an unexpected heterogeneity of infections by Chlamydia pecorum. J Clin Microbiol. 2015;53(5):1573-81.

18. Hong EE, Okitsu CY, Smith AD, Hsieh CL. Regionally specific and genomewide analyses conclusively demonstrate the absence of CpG methylation in human mitochondrial DNA. Mol Cell Biol. 2013;33(14):2683-90.

19. Pillonel T, Bertelli C, Salamin N, Greub G. Taxogenomics of the order Chlamydiales. International Journal of Systematic and Evolutionary Int J Syst Evol Microbiology. 2015;65(4):1381-93.

20. Rockey DD. Unraveling the basic biology and clinical significance of the chlamydial plasmid. J Exp Med. 2011;208(11):2159-62.

21. Song L, Carlson JH, Whitmire WM, Kari L, Virtaneva K, Sturdevant DE, Watkins H, Zhou B, Sturdevant GL, Porcella SF, et al. Chlamydia trachomatis plasmid-encoded Pgp4 is a transcriptional regulator of virulence-associated genes. Infect Immun. 2013;81(3):636-44.

22. Yang C, Starr T, Song L, Carlson JH, Sturdevant GL, Beare PA, Whitmire WM, Caldwell HD. Chlamydial Lytic Exit from Host Cells Is Plasmid Regulated. MBio. 2015;6(6):e01648-01615.

23. Pickett MA, Everson JS, Pead PJ, Clarke IN. The plasmids of Chlamydia trachomatis and Chlamydophila pneumoniae (N16): accurate determination of copy number and the paradoxical effect of plasmid-curing agents. Microbiol. 2005;151(Pt 3):893-903. 
24. Ferreira R, Borges V, Nunes A, Borrego MJ, Gomes JP. Assessment of the load and transcriptional dynamics of Chlamydia trachomatis plasmid according to strains' tissue tropism. Microbiol Res. 2013;168(6):333-9.

25. Jelocnik M, Bachmann NL, Kaltenboeck B, Waugh C, Woolford L, Speight KN, Gillett A, Higgins DP, Flanagan C, Myers GS, et al. Genetic diversity in the plasticity zone and the presence of the chlamydial plasmid differentiates Chlamydia pecorum strains from pigs, sheep, cattle, and koalas. BMC Genomics. 2015;16:893.

26. Kalman S, Mitchell W, Marathe R, Lammel C, Fan J, Hyman RW, Olinger L, Grimwood J, Davis RW, Stephens RS. Comparative genomes of Chlamydia pneumoniae and C. trachomatis. Nat Genet. 1999;21(4):385-9.

27. Yilmaz S, Allgaier M, Hugenholtz P. Multiple displacement amplification compromises quantitative analysis of metagenomes. Nat Methods. 2010; 7(12):943-4.

28. Vasilevsky S, Stojanov M, Greub G, Baud D. Chlamydial polymorphic membrane proteins: regulation, function and potential vaccine candidates. Virulence. 2016;7(1):11-22.

29. Henderson IR, Lam AC. Polymorphic proteins of Chlamydia spp.autotransporters beyond the Proteobacteria. Trends Microbiol. 2001;9(12):573-8.

30. Collingro A, Tischler P, Weinmaier T, Penz T, Heinz E, Brunham RC, Read TD, Bavoil PM, Sachse K, Kahane S, et al. Unity in variety-the pan-genome of the Chlamydiae. Mol Biol Evol. 2011;28(12):3253-70.

31. Chen D, Lei L, Lu C, Flores R, DeLisa MP, Roberts TC, Romesberg FE, Zhong G. Secretion of the chlamydial virulence factor CPAF requires the Sec-dependent pathway. Microbiol. 2010;156(10):3031-40.

32. Lutter El, Martens C, Hackstadt T. Evolution and conservation of predicted inclusion membrane proteins in chlamydiae. Comp Funct Genomics. 2012; 2012:362104.

33. Mital J, Miller NJ, Dorward DW, Dooley CA, Hackstadt T. Role for chlamydial inclusion membrane proteins in inclusion membrane structure and biogenesis. PloS One. 2013;8(5):e63426.

34. Podar M, Abulencia CB, Walcher M, Hutchison D, Zengler K, Garcia JA, Holland T, Cotton D, Hauser L, Keller M. Targeted access to the genomes of low-abundance organisms in complex microbial communities. Appl Environ Microbiol. 2007;73(10):3205-14

35. Bolger AM, Lohse M, Usadel B. Trimmomatic: a flexible trimmer for Illumina sequence data. Bioinformatics. 2014;30(15):2114-20.

36. Bankevich A, Nurk S, Antipov D, Gurevich AA, Dvorkin M, Kulikov AS, Lesin VM, Nikolenko SI, Pham S, Prjibelski AD, et al. SPAdes: a new genome assembly algorithm and its applications to single-cell sequencing. J Comput Biol. 2012; 19(5):455-77.

37. Gurevich A, Saveliev V, Vyahhi N, Tesler G. QUAST: quality assessment tool for genome assemblies. Bioinformatics. 2013;29(8):1072-5.

38. Aziz RK, Bartels D, Best AA, DeJongh M, Disz T, Edwards RA, Formsma K, Gerdes S, Glass EM, Kubal M, et al. The RAST Server: rapid annotations using subsystems technology. BMC Genomics. 2008;9:75.

39. Rutherford K, Parkhill J, Crook J, Horsnell T, Rice P, Rajandream MA, Barrell B. Artemis: sequence visualization and annotation. Bioinformatics. 2000;16(10):944-5.

40. Bengtsson J, Eriksson KM, Hartmann M, Wang Z, Shenoy BD, Grelet GA, Abarenkov K, Petri A, Rosenblad MA, Nilsson RH. Metaxa: a software tool for automated detection and discrimination among ribosomal small subunit (12S/16S/18S) sequences of archaea, bacteria, eukaryotes, mitochondria, and chloroplasts in metagenomes and environmental sequencing datasets. Antonie van Leeuwenhoek. 2011;100(3):471-5.

41. Kearse M, Moir R, Wilson A, Stones-Havas S, Cheung M, Sturrock S, Buxton S, Cooper A, Markowitz S, Duran C, et al. Geneious Basic: an integrated and extendable desktop software platform for the organization and analysis of sequence data. Bioinformatics. 2012;28(12):1647-9.

42. Li H, Durbin R. Fast and accurate long-read alignment with BurrowsWheeler transform. Bioinformatics. 2010;26(5):589-95.

43. Quinlan AR, Hall IM. BEDTools: a flexible suite of utilities for comparing genomic features. Bioinformatics. 2010;26(6):841-2.

44. Sullivan MJ, Petty NK, Beatson SA. Easyfig: a genome comparison visualizer. Bioinformatics. 2011;27(7):1009-10.

45. Sahl JW, Caporaso JG, Rasko DA, Keim P. The large-scale blast score ratio (LS-BSR) pipeline: a method to rapidly compare genetic content between bacterial genomes. Peer J. 2014;2:e332.
46. Price MN, Dehal PS, Arkin AP. FastTree: computing large minimum evolution trees with profiles instead of a distance matrix. Mol Biol Evol. 2009;26(7):1641-50

47. Krogh A, Larsson B, von Heijne G, Sonnhammer EL. Predicting transmembrane protein topology with a hidden Markov model: application to complete genomes. J Mol Biol. 2001;305(3):567-80.

\section{Submit your next manuscript to BioMed Central and we will help you at every step:}

- We accept pre-submission inquiries

- Our selector tool helps you to find the most relevant journal

- We provide round the clock customer support

- Convenient online submission

- Thorough peer review

- Inclusion in PubMed and all major indexing services

- Maximum visibility for your research

Submit your manuscript at www.biomedcentral.com/submit

) Biomed Central 